\title{
Central mucoepidermoid carcinoma of the mandible associated with Systemic lupus erythematosus
}

\author{
Hari Ram ${ }^{1}$, Isha Atam ${ }^{2}$, Debraj Howlader ${ }^{3}$, Satish Kumar ${ }^{\star, \dagger, 4}$, Madhu Kumar $^{5}$ \\ ${ }^{1}$ Professor, Department of Oral and Maxillofacial Surgery, King George's Medical University Lucknow, India. \\ ${ }^{2}$ Intern, King george's medical University Lucknow. \\ ${ }^{3}$ Senior Resident Department of Oral and Maxillofacial Surgery, King George's Medical University Lucknow, \\ India \\ ${ }_{4}^{4}$ Senior resident, Department of Medicine, King george's medical University, Lucknow. \\ ${ }^{5}$ Professor, Department of Pathology King George's Medical University Lucknow, India.
}

DDOI: https://doi.org/10.15520/jcmro.v2i10.222

Accepted 21-10-2019; Received 02-10-2019; Publish Online 23-10-2019

\author{
Reviewed By: \\ Dr. SABINA KHANAM \\ Department: \\ Reviewer/CMRO
}

\begin{abstract}
Mucoepidermoid carcinoma of the salivary gland is the "most common salivary gland tumor. This tumour inhabits mainly parotid, palatal minor salivary glands and the submandibular salivary glands. Central involvement of the mandible or maxilla is very rare. Here we report a case of central mucoepidermoid carcinoma associated with systemic lupus erythematous in a 25 year old female patient, who was managed by surgery.
\end{abstract}

\section{INTRODUCTION:}

Mucoepidermoid carcinoma is the commonest malignant salivary gland tumour. It mainly involves the parotid, palatal minor salivary glands and the submandibular salivary glands [1]. Central involvement of the mandible or maxilla is known as central mucoepidermoid carcinoma (CMEC) which is very uncommon and representing only about $2-4 \%$ of all mucoepidermoid carcinomas[[2]. There are many theories of etiopathogenesis of these lesions. The most appropriate theories are (1) embryonic or iatrogenic entrapment of salivary tissue in bone and (2) salivary metaplasia of odontogenic cyst epithelium [3]. Due to the rarity of the condition, literature on this distinct entity exists mostly as case reports. The risk factors and conditions associated with this disease remain unclear. The main stay of treatment is en bloc resection or hemimandibulectomy.

\section{CASE PRESENTATION:}

A 25 year old female reported to the Department of Oral and Maxillofacial Surgery with the chief complaint of a slow growing, painless swelling in the left lower jaw since 2 years. Patient had a history of pulmonary tuberculosis which was treated 8 years ago. Systemic examination was unremarkable. History of photosensitivity was also present. Extra oral

* Corresponding author.

$\dagger$ Email: dr.satishkgmu@gmail.com inspection revealed a diffuse swelling measuring 3X3 centimeter at the left side of body of mandible along with a butterfly rash over the malar region Figure 1.

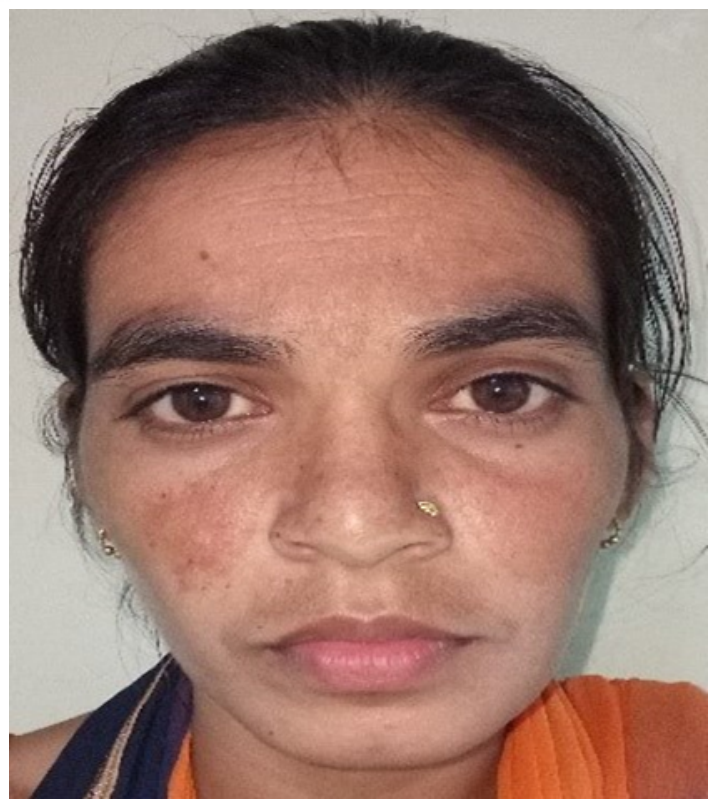

Figure 1. Preoperative photograph showing slight swelling at left side of mandible and butter fly rash on face.

On palpation swelling was hard and non-tender. On Intraoral inspection full complements of teeth were present 
and a bony expansion in the left body region was appreciable.

A Computed Tomography (CT) scan of the face and neck revealed a $3 \mathrm{X} 3 \mathrm{X} 2.5 \mathrm{~cm}$ osteoexpansile and osteodestructive lesion of the left body of mandible from canine to molar area involving the neurovascular bundle. There was an impacted third molar along with multiple specks /septae of calcification within the lesion along with bicortical expansion and perforation. Non-specific lymphadenopathy was noted in bilateral $1 \mathrm{~b}$ regionFigure 2 .

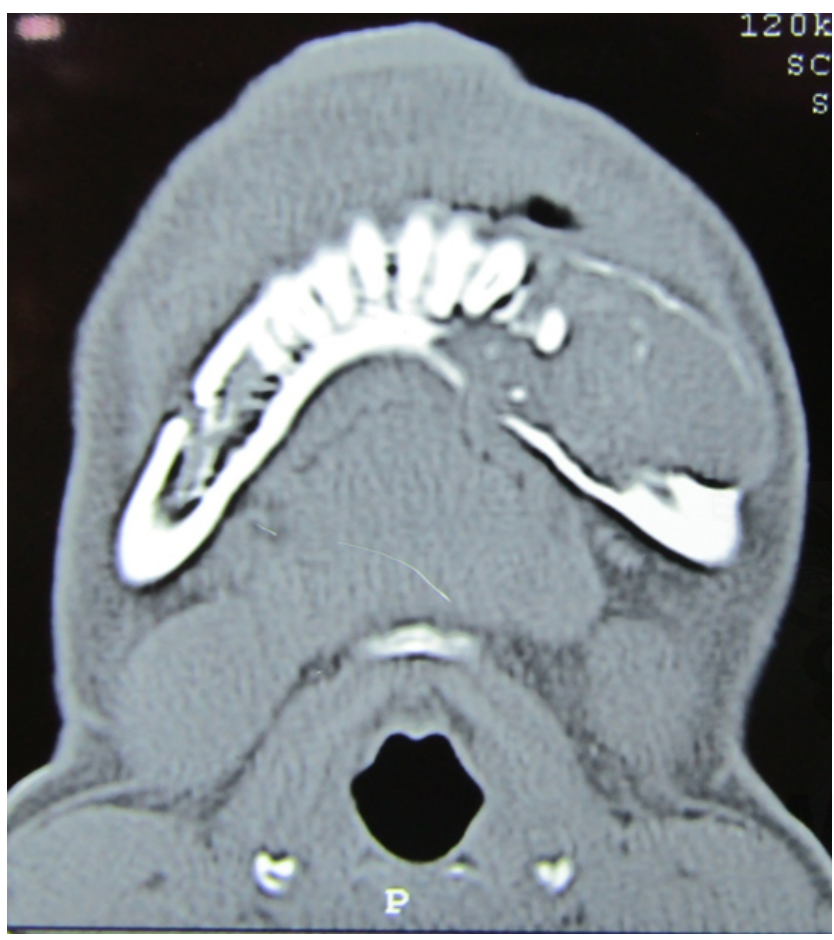

Figure 2. CT-Axial photograph mandible showing bone destruction on left side of mandible

Butterfly rash was present on malar region and history of photosensitivity was also noted. Antinuclear antibody test (ANA) was done, which was positive. Anti ds-DNA was also positive.

Biopsy was done from the buccal vestibule. Histopathologic examination revealed haphazardly distributed mucin filled cystic spaces and irregular tumor nests containing mucoid, squamoid and intermediate cells. The stroma showed extravasated pools of mucin with mild chronic inflammatory infiltrates. The mucoid cells showed abundant mucin and forming glandular pattern. The intermediate cells were polygonal in shape with bland nuclear chromatin and scant cytoplasm. The squamoid cells were pleomorphic with high nuclear-cytoplasmic ratio,hyperchromatic nucleus with inconspicuous nucleoli and mild to moderate amount of cytoplasm. The findings were suggestive of mucoepidermoid carcinoma Figures 3 and 4.

The course of SLE in this case was monophasic as there was no any other organ involvement and disease presentation except mucoepidermoid carcinoma.

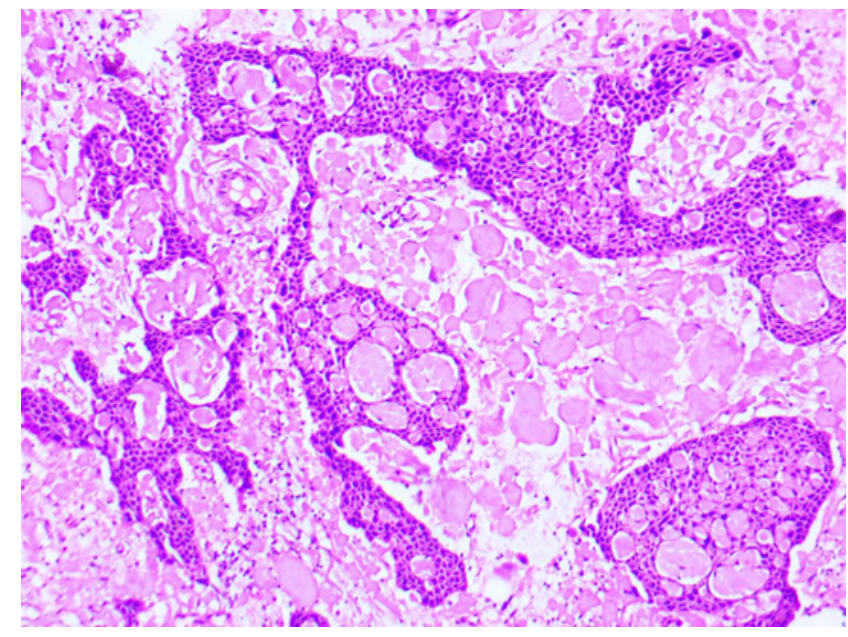

Figure 3. Section showing sheets and nest of malignant ductal cells with mixed glandular, squamous and intermediate cells in the background of extracellular mucin (H\&E Stain,X10).

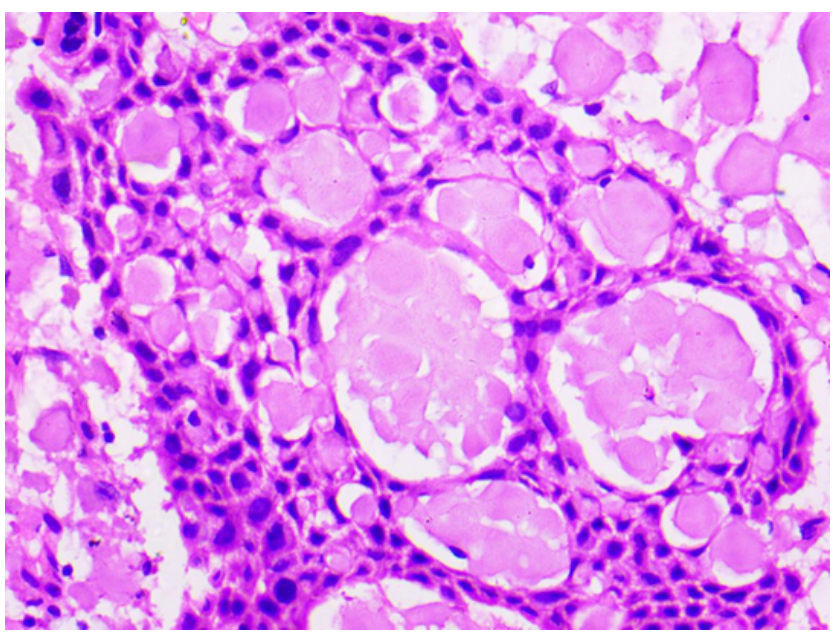

Figure 4. Section showing multiple cystic spaces lined by mucin producing cells with polygonal intermediate cells intermixed with squamoid cells without significant keratinisation (H\&E Stain, $\mathrm{x} 40$ ).

Based on the radiological and histopathological findings, segmental mandibulectomy from the angle of the left side to lateral incisor of right side was done (the anterior margin of resection was extended based on intra operative finding of marrow infiltration beyond radiographic margin)Figure 5 . Bony reconstruction was not done as the patient was unwilling for further surgical morbidity of the donor site. A titanium reconstruction plate was fixed to maintain facial contour and prevent mandibular deviation and associated sequel of hemi-mandibulectomy Figures 6 and 7 .

Patient improved well and was disease free at 1year of follow up. No recurrence was seenFigure 8. 


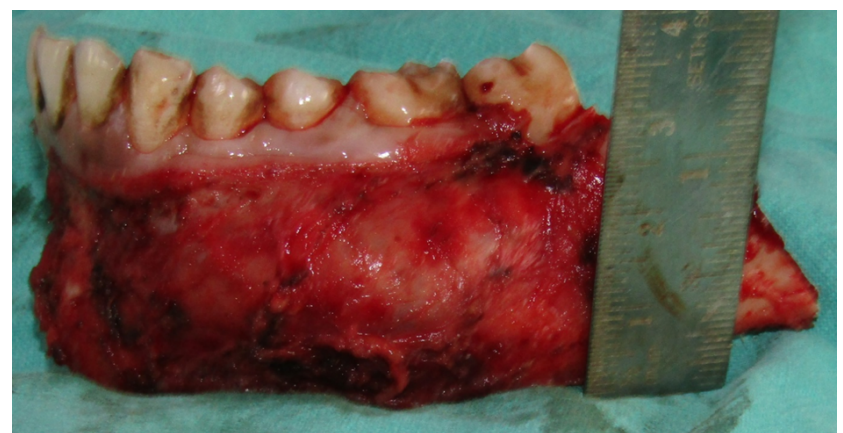

Figure 5. Resected specimen

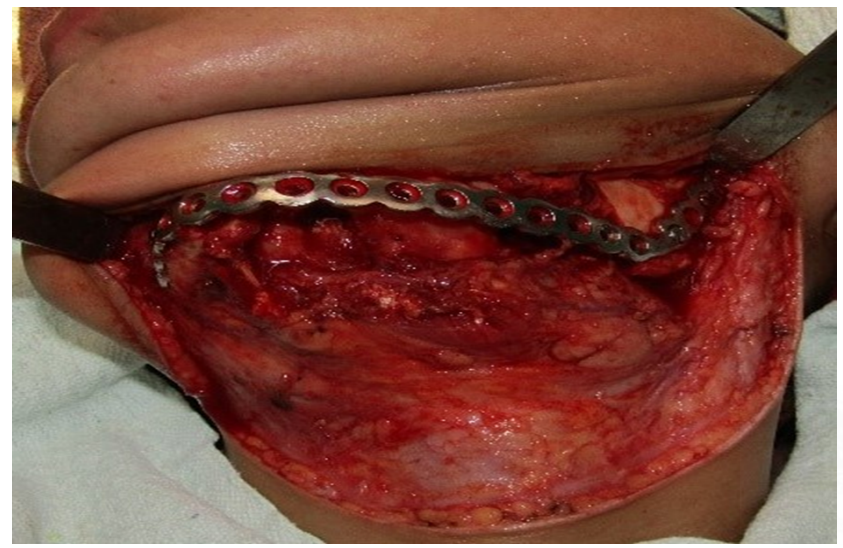

Figure 6. Post operative photograph reconstructed with bone plate

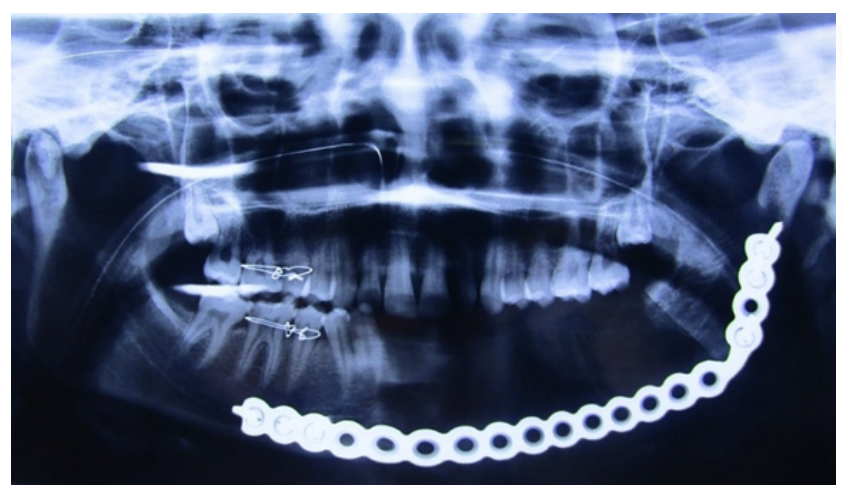

Figure 7. Post operative Orthopantomogram

\section{DISCUSSION:}

The incidence of CMEC in women is almost twice compared to males. Eversole et.al reported that roughly $50 \%$ of mandibular CEMECs were associated with dental cysts and/or impacted teeth [3]. This finding was consistent with our case. Additionally the clinical, radiologic and histological findings of the lesion satisfied the diagnostic criteria of CMEC [4].

The biologic behaviour and prognosis of CMEC correlates with the histologic grading with low grade tumours having more favourable prognosis $[1,2,5]$. Additionally younger age is considered a favourable prognostic factor [6]. Based on

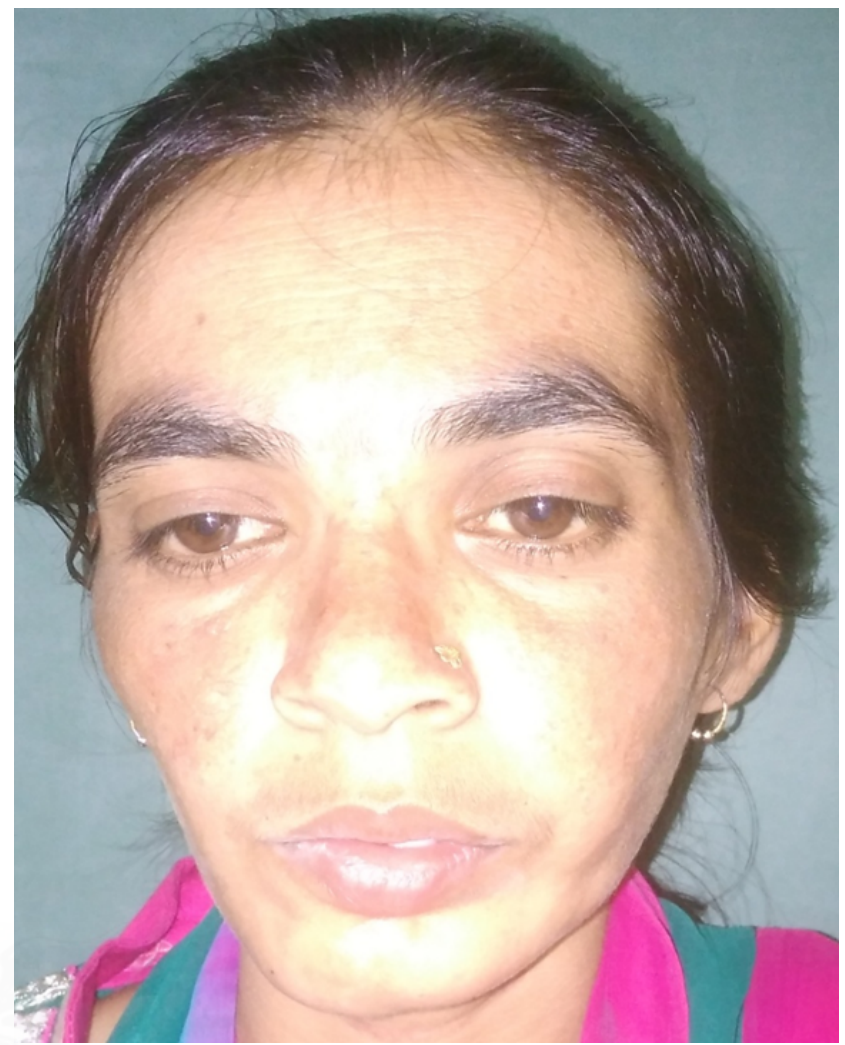

Figure 8. Postoperative photograph of patient

the histologic findings this case was classified as low grade CMEC [1]. Fortunately most CMEC usually are low grade with fair prognosis having an overall survival rate of $95.1 \%$ and disease free survival rate of $87.5 \%$ as reported by Ozawa et. $\mathrm{Al}[[6]$.

Optimal management of CMEC is en bloc resection or hemimandibulectomy. Conservative approaches cause recurrence. Brookstone and Huvos reported recurrence rate of $40 \%$ with conservative approaches such as marsupialisation, curettage and enucleation while only $4 \%$ recurrence was observed with aggressive treatment such as segmental resection with or without neck dissection and advujant therapy [7]. Neck dissection is done in case of nodal involvement $[1,2,4,8]$.

Although MEC is considered to be radio resistant, postoperative radiotherapy has been shown to improve loco-regional control [6]. Adjuvant Radiotherapy is recommended in the case of close or positive margins and in intermediate to high grade tumours [1, 2, 4, 6-9].

Keeping in mind the tendency of perineural invasion of the tumour resection margin was extended to $2.5 \mathrm{~cm}$ beyond the radiographic margin and the anterior margin further advanced intra-operatively based on the suspicion of probable marrow invasion. The suspicion was confirmed by the final histopathology report which revealed disease $2.5 \mathrm{~cm}$ from the posterior margin but only $2 \mathrm{~cm}$ from anterior margin. All margins of the final specimen measuring $8 \mathrm{~cm} \times 3 \mathrm{~cm} \times 2 \mathrm{~cm}$ were tumour free with no evidence of perineural invasion and only reactive hyperplasia of the Level I nodes. 
In addition to the rarity of CMEC the peculiarity of this case lies in its association with SLE.

Over the past few decades it has become increasingly clear that patients with SLE are known to have an increased risk of head and neck cancers. However it is also possible that SLE and a cancer presenting together represent lupus as a paraneoplastic manifestation. Specifically, lupus patients have higher risk of lymphoma and other cancers, like, cancer of the cervix. Researchers have elucidated certain connections between lupus and cancer [[10].

Learning Points:

1. Central mucoepidermoid carcinoma (CMEC) is very uncommon malignant tumour.

2. CMEC associated with SLE have not been reported in literature. Probably it is first reported case.

3. Patients having SLE have increased risk of cancers.

4. Surgical resection is the main stay of treatment.

\section{REFERENCES}

[1].Marx, Robert E., and Diane Stern. Oral and Maxillofacial Pathology: a Rationale for Diagnosis and Treatment. Quintessence Pub., 2012.

[2].F. R. Pires, O. Paes de Almeida, M. A. Lopes, D. Elias da Cruz Perez, L. P. Kowalski: Central mucoepidermoid carcinoma of the mandible: report of four cases with long-term follow-up. Int. J. Oral Maxillofac. Surg. 2003; 32: 378-382. [3].Eversole LR Mucoepidermoid Carcinoma: Review of 815 reported cases. Oral Surg Oral Med Oral Pathol 1970, 28:490-495

[4].Simon D, Somanathan T, Ramdas K, Pandey M. Central mucoepidermoid carcinoma of mandible - a case report and review of the literature. World J Surg Oncol 2003;25:1. [5].Liu S, et al: Prognostic factors in primary salivary gland mucoepidermoid carcinoma: an analysis of 376 cases in an Eastern Chinese population. Int J Oral Maxillofac Surg 2014;43:667-673.

[6].Hiroyuki Ozawa, Toshiki Tomita, Koji Sakamoto, Takamasa Tagawa, Ryoichi Fujii, Sho Kanzaki, Kaoru Ogawa, Kaori Kameyama, Masato Fujii. Mucoepidermoid Carcinoma of the Head and Neck: Clinical Analysis of 43 Patients. Jpn J Clin Oncol 2008;38(6)414-418.

[7].Brookstone MS and Huvos AG Central salivary gland tumors of the maxilla and mandible: a clinicopathologic study of 11 cases with an analysis of the literature. J Oral Maxillofac Surg $1992,50: 229-236$

[8].Sepúlveda et al.: Mandibular Central Mucoepidermoid Carcinoma: A Case Report and Review of the Literature. Case Rep Oncol 2014;7:732-738

[9].F.V. de Mello-Filho et al. Central mucoepidermoid carcinoma: Report of 2 cases. British Journal of Oral and Maxillofacial Surgery 46 (2008) 239-241

[10].Gayed M, Bernatsky S, Ramsey-Goldman R, Clarke AE, Gordon C. Lupus and cancer. Lupus. 2009 May;18(6):479-85. 